Polymer Journal, Vol. 39, No. 2, pp. 181-186 (2007)

(C) 2006 The Society of Polymer Science, Japan

\title{
Microwaves Determine the Orientational Distribution of Collagen Fibers in a Whole Cobra Skin
}

\author{
Katsunori NiITSUmA, ${ }^{1}$ Sachiko MiYAGAwA, ${ }^{1}$ and Shigeyoshi OSAKI ${ }^{2, \dagger}$ \\ ${ }^{1}$ Department of Dermatology, Faculty of Medicine, Nara Medical University, Kashihara, Nara 634-8521, Japan \\ ${ }^{2}$ Department of Chemistry, Faculty of Medicine, Nara Medical University, Kashihara, Nara 634-8521, Japan
}

(Received October 6, 2006; Accepted November 16, 2006; Published December 27, 2006)

\begin{abstract}
The orientation of collagen fibers was determined in a small region of a cobra skin using Osaki's microwave method, and compared with the mechanical breaking method. The microwave method provided the orientational distribution of collagen fibers in the whole cobra skin. The collagen fibers were, on average, aligned almost parallel to the direction of the cranial to caudal end in the dorsum of the trunk and caudal regions, while they were aligned randomly in the abdomen. The degree of collagen-fiber orientation decreased gradually from the dorsum to the abdomen, and was relatively small in the cranial region, compared with that in the other dorsum regions. The results obtained using Osaki's microwave method indicate that the orientational distribution of collagen fibers in cobra skin is closely related to the style of functional motion, such as shrinkage and expansion, of the cobra.

[doi:10.1295/polymj.PJ2006127]

KEY WORDS Cobra Skin / Collagen Fiber / Orientation / Motion / Microwaves / Anisotropy /
\end{abstract}

The determination of collagen-fiber orientation constituting biological supporting tissues, such as skin and bone, is essential for understanding the relationship between the mechanical properties and the fine structure of tissues. Conventional methods, such as $\mathrm{X}$-ray diffraction and electron microscopy have been used to determine the collagen-fiber orientation in biological tissues, such as the bovine aortic wall ${ }^{1}$ and bovine bone. ${ }^{2}$ These methods are not practical for determining collagen-fiber orientation in all biological tissues because the measurements are restricted to a very small region and are time-consuming. Although there have been reports on the stress-strain relationships within human skin, ${ }^{3}$ along with the mechanical properties of rat $\operatorname{skin}^{4}$ and human skin, ${ }^{5}$ there have been few reports on the mechanical properties of supporting tissues in relation to collagen-fiber orientation. ${ }^{6,7}$

The orientation of collagen fibers affects the mechanical properties of the skin related to animal motion; however, few reports have addressed fiber orientation from the viewpoint of the functional motion of the skin. Snake skin may be an appropriate material to clarify the relationship between collagen-fiber orientation and mechanical anisotropy, since the motion of a snake is comparatively simple. In particular, identifying the mechanical properties of the skin is very important for understanding the actual motion of snake skin, even though a large amount of time is required for mechanical measurements. In the preceding paper, ${ }^{8}$ the mechanical properties of snake skin were reported; however, it was difficult to obtain information on fiber orientation in a restricted region of snake skin since a number of strip samples were prepared from a wide region to measure the angular dependence of the mechanical breaking strength, which only gave rough data of mechanical anisotropy in the snake skin.

Previously, one of the authors developed a method to rapidly determine the fiber orientation of sheets of paper, ${ }^{9}$ nonwoven fabrics, ${ }^{10}$ and the collagen-fiber orientation for human blood vessels, ${ }^{11}$ calf leather, ${ }^{12,13}$ snake leather ${ }^{14}$ and human bone ${ }^{15,16}$ using Osaki's microwave method. ${ }^{17-19}$ Thus, Osaki's microwave method has enabled us to measure fiber orientation even in a restricted region of snake skin.

The present paper describes the use of Osaki's microwave method to determine fiber orientation, even in a restricted region, and its application to cobra skin to determine the orientational distribution of collagen fibers in the whole skin. This provides important information for understanding the functional motion of the skin.

\section{EXPERIMENTAL}

\section{Preparation of Samples from Cobra Skin}

A sheet of cobra (Najanaja kaouthia) leather was prepared as follows. The whole cobra skin was cut along the middle of the abdomen from the caudal end in a cranial direction (CCD). The skin was then stripped away from the body and pickled in salt. After the salt had been removed with water, the skin was soaked in hydrated lime. It was subsequently removed, pickled in acid, resin-treated, stretched on a board and

${ }^{\dagger}$ To whom correspondence should be addressed (Tel/Fax: +81-744-29-8810; E-mail: s-osaki@ naramed-u.ac.jp). 


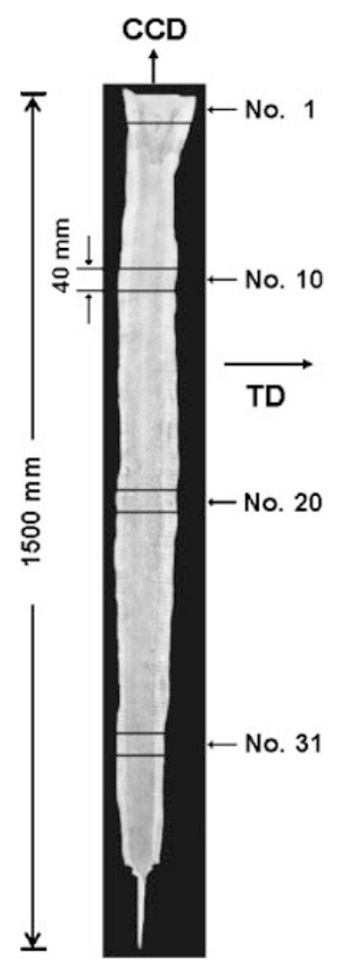

Figure 1. A leather sheet, approximately $0.3 \mathrm{~mm}$ thick, max. $80 \mathrm{~mm}$ wide and $1500 \mathrm{~mm}$ long with a $210 \mathrm{~g} / \mathrm{m}^{2}$ basis weight, was prepared by cutting along the center of the abdomen in the CCD (from the caudal to cranial end) of cobra skin. The sheet was cut in the transverse direction (TD) and test samples $40 \mathrm{~mm}$ long were prepared. The samples were designated as Nos. 1, 10, 20 , and 31 with the changing position in the CCD.

allowed to dry at room temperature. Finally, the skin was made into a leather sheet of $c a .1500 \mathrm{~mm}$ (length) $\times c a .80 \mathrm{~mm}$ (width) $\times c a .0 .30 \mathrm{~mm}$ (thickness), as shown in Figure 1. The basis weight of the leather sheet was $c a .210 \mathrm{~g} / \mathrm{m}^{2}$. Samples used as test materials were prepared by cutting the cobra leather into appropriate sizes for mechanical and microwave measurements. Many treatments used in the process of conversion were chemically weak, much different from chemically strong treatments with strong acids and bases. Binding between the scales and textures of the leather was extremely strong, even though stretching force was applied to the skin. Thus, the collagen fibers in the skin were so strongly intertwined that they did not undergo significant changes in the fine structures related to fiber orientation when converted into leather via the process described above.

The sheet was cut in the transverse direction (TD), perpendicular to the CCD, and test samples of $40 \mathrm{~mm}$ were prepared (see Figure 1). The samples were designated as No. 1, No. 10, No. 20 and No. 31, according to the changing position of the CCD of the cobra leather. Samples No. 1, 10, 20, and 31 were prepared from parts of the cranial, trunk and caudal regions, respectively.

\section{Microwave Measurements}

Each test sample $(40 \mathrm{~mm}$ length of CCD $\times$ whole width of TD) was inserted into a narrow gap between a pair of waveguides constituting a cavity resonator system, and was rotated around the axis central to the sample plane. Microwaves of $12 \mathrm{GHz}$ were irradiated in this plane, and the transmitted microwave intensity was measured at different angles of rotation..$^{9,10,17,19,20}$ Such measurements were carried out in different positions by shifting the position of the sample discretely at intervals of $2.5 \mathrm{~mm}$ along the TD. Since the transmitted microwaves reflected the interaction between the polarized microwaves and the dipole moments in macromolecules constituting the sample, ${ }^{9,10,17-20}$ the angular dependence of the transmitted microwave intensity, called the orientation pattern, reflects the distribution of the molecular or fiber orientation, giving the orientation angle $(\beta)$ and the degree of fiber orientation (DFO). ${ }^{13,17,19}$ Thus, the microwave method mainly reflects the orientation of collagen fibers contained in the calf and cobra skins. ${ }^{13,14}$ The direction giving the minimum transmitted microwave intensity was designated as $\beta,{ }^{17,19}$ corresponding to the angle between the main axis of the collagen fibers and the CCD. The maximum-to-minimum ratio of the transmitted microwave intensity was defined as DFO, which increased with the increasing preferential alignment of fibers. ${ }^{13}$ Since a DFO of 1.0 reflects random orientation, the value of (DFO -1.0), which is zero or positive, reflects the net degree of orientation. The $\mathrm{MW}_{(\mathrm{TD} / \mathrm{CC})}$ is the ratio of transmitted microwave intensity in TD to CCD, representing anisotropy in TD to CCD.

The resonance curve before and after insertion of a sample into the cavity resonator system was expressed in terms of transmitted microwave intensity: $9,12,17,19$

$$
I(\theta)=I_{\mathrm{i} 0}(\theta) /\left\{1+Q_{\mathrm{i}}^{2}(\theta)\left[f_{\mathrm{i} 0}(\theta) / f-f / f_{\mathrm{i} 0}(\theta)\right]^{2}\right\}
$$

Here, $I, I_{\mathrm{i} 0}$ and $Q_{\mathrm{i}}(\mathrm{i}=1,2)$, are the transmitted microwave intensities at a given frequency $f$, its value at resonance frequency $f_{\mathrm{i} 0}$ and the ratio of the resonance frequency to one-half of the width of $\Delta f$ in the resonance curve, respectively, all at a rotation angle of $\theta$.

Subscripts 1 and 2 indicate the values before and after the insertion of a test sample. In the present experiments, the angular dependence of transmitted microwave intensity in the resonance curve was measured at a fixed frequency, where the intensity was just half the peak intensity, higher than the response frequency. ${ }^{17,19}$ Errors in the values observed for the orientation angle and the degree of orientation measured using Osaki's microwave method were within $\pm 1^{\circ}$ and $\pm 5 \%$, respectively. Errors in the orientation angle depend on variations of the 


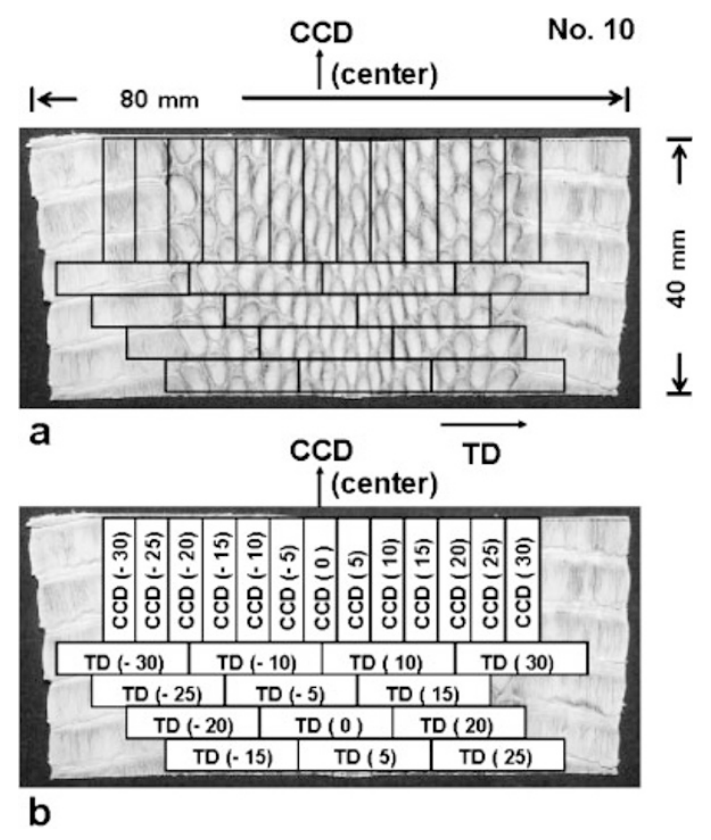

Figure 2. a: 26 strip specimens $(5 \mathrm{~mm} \times 20 \mathrm{~mm})$ were prepared by cutting sample No. 10 in CCD and TD for mechanical measurements. b: Notation of each specimen. Strip specimens prepared by cutting in the $\mathrm{CCD}$ and TD were designated as CCD (M) and TD (M). M indicates the distance ( $\mathrm{mm}$ ) measured from the center of sample No. $10(+$ means the right side from the center of the sample).

sample axis to the CCD, while errors in the degree of orientation also depend on variations in the sample thickness.

\section{Mechanical Measurements}

Twenty six strip specimens $(5 \mathrm{~mm}$ wide $\times 20 \mathrm{~mm}$ long) were prepared by cutting sample No. 10 in CCD and TD, as shown in Figure 2. Here, strip specimens were designated as CCD (M) and TD (M), respectively, as shown in Figure 2. M indicates the distance in $\mathrm{mm}$ measured from the central position of sample No. 10 ( + in $\mathrm{M}$ means the right side from the central position of the sample). For example, -30 (left) and 30 (right) for $\mathrm{M}$ correspond to the positions around the abdomen, whereas 0 (center) for $\mathrm{M}$ corresponds to the central position around the dorsum. Force-elongation curves were measured with a stretching velocity of $20 \mathrm{~mm} / \mathrm{min}$ using an RTC1150A universal testing machine (Orientec, Tokyo, Japan). The mechanical breaking strength (BS), defined as the strength at the breaking point, was determined for each specimen.

\section{RESULTS}

\section{The Ratio of BS in CCD to TD}

Since the angular dependence of mechanical breaking strength requires a number of strip samples, as

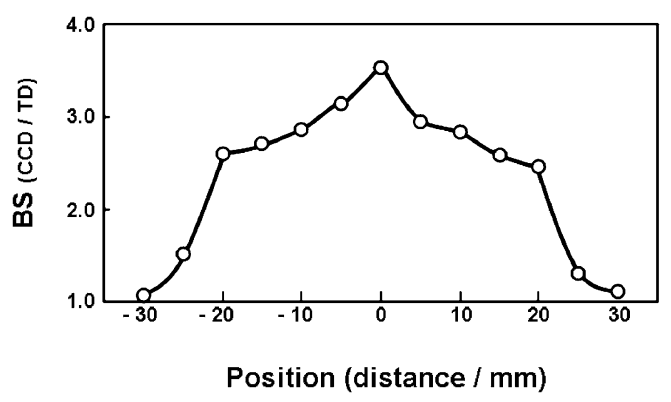

Figure 3. $\mathrm{BS}_{(\mathrm{CCD} / \mathrm{TD})}$, the ratio of $\mathrm{BS}$ in $\mathrm{CCD}$ to $\mathrm{TD}$, is plotted against the position in TD. The specimens used for mechanical measurements of BS were prepared from sample No. 10.

shown in the preceding paper, it gives only the average data over a wide region for mechanical anisotropy. Thus, we have to estimate mechanical anisotropy in a relatively small region. The ratio, $\mathrm{BS}_{(\mathrm{CCD} / \mathrm{TD})}$, of $\mathrm{BS}$ in the two perpendicular directions of CCD and TD was used as the index for estimating mechanical anisotropy.

$\mathrm{BS}_{(\mathrm{CCD} / \mathrm{TD})}$ is plotted in Figure 3 against the position in $\mathrm{TD} . \mathrm{BS}_{(\mathrm{CCD} / \mathrm{TD})}$ was largest in the center of the dorsum and decreased from 3.51 to 1.06 , with the change in the position from the dorsum to the abdomen. This indicates that the leather was mechanically stronger around the dorsum in CCD than in TD. The difference in mechanical strength between CCD and TD was small around the abdomen. The results mean that mechanical anisotropy increases with the changing position of the abdomen to the dorsum, however, strictly speaking, $\mathrm{BS}_{(\mathrm{CCD} / \mathrm{TD})}$ does not necessarily reflect mechanical anisotropy in the same small region, since the strip samples in the CCD and TD occupy slightly different positions, as shown in Figure 2.

\section{Comparison of Mechanical Anisotropy with Micro- wave Anisotropy}

In order to understand the functional motion of cobra skin, it is necessary to measure mechanical anisotropy in as small a region as possible; however, this is very difficult in cobra leather using the mechanical method since it gives only an average value over a relatively wide region. ${ }^{8}$ However, we have recently overcome such difficulties by using microwave measurements and made it possible to measure fiber orientation by shifting the sheet sample by intervals of $25 \mathrm{~mm}$.

Here, we tried to ascertain whether it is possible to use Osaki's microwave method ${ }^{15,17}$ to evaluate fiber orientation instead of the measurements of mechanical anisotropy. First, we have to clarify the relationship between mechanical anisotropy and fiber orientation as determined by Osaki's microwave method. Me- 


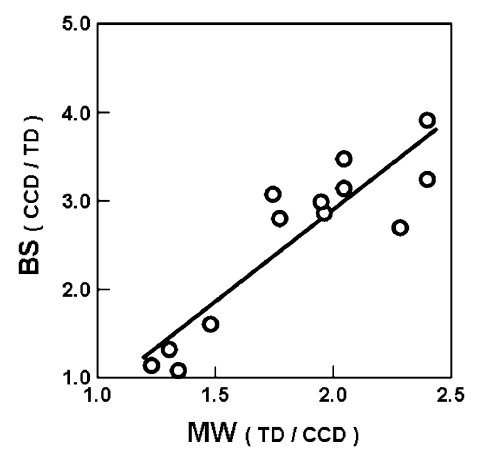

Figure 4. Comparison of $\mathrm{BS}_{(\mathrm{CCD} / \mathrm{TD})}$ with $\mathrm{MW}_{(\mathrm{TD} / \mathrm{CCD})}$. $\mathrm{BS}_{(\mathrm{CCD} / \mathrm{TD})}$ was plotted against $\mathrm{MW}_{(\mathrm{TD} / \mathrm{CCD})}$. The empirical equation was obtained as $\mathrm{BS}_{(\mathrm{CCD} / \mathrm{TD})}=-1.212+2.076 \mathrm{MW}_{(\mathrm{TD} / \mathrm{CCD})}$ with a correlation coefficient of $0.89 . \mathrm{MW}_{(\mathrm{TD} / \mathrm{CCD})}$ was defined as the ratio of transmitted microwave intensity in TD to CCD. $\mathrm{MW}_{(\mathrm{TD} / \mathrm{CCD})}$ and $\mathrm{BS}_{(\mathrm{CCD} / \mathrm{TD})}$ were determined, respectively, for sample No. 10 and strip specimens prepared from sample No. 10, and were compared at the same position of TD.

chanical anisotropy $\mathrm{BS}_{(\mathrm{CCD} / \mathrm{TD})}$ was determined, and then compared with the anisotropy of $\mathrm{MW}_{(\mathrm{TD} / \mathrm{CCD})}$, which was defined as the ratio of transmitted microwave intensity in TD to CCD.

Figure 4 shows the relationship between $\mathrm{BS}_{(\mathrm{CCD} / \mathrm{TD})}$ and $\mathrm{MW}_{(\mathrm{TD} / \mathrm{CCD})}$. Even though the data were scattered, the empirical equation was obtained as

$$
B S_{(\mathrm{CCD} / \mathrm{TD})}=-1.212+2.076 \mathrm{MW} \text { (TD/CCD) }
$$

with a correlation coefficient of 0.89 . Here, mechanical breaking strength for strip specimens was measured after microwave measurements for sample No. 10.

It was found that $\mathrm{BS}_{(\mathrm{CCD} / \mathrm{TD})}$ was roughly proportional to $\mathrm{MW}_{(\mathrm{TD} / \mathrm{CCD})}$. Thus, $\mathrm{MW}_{(\mathrm{TD} / \mathrm{CCD})}$ can be used as the index reflecting anisotropy instead of mechanical anisotropy of $\mathrm{BS}_{(\mathrm{CCD} / \mathrm{TD})}$. In previous papers, ${ }^{8,14}$ electron scanning microphotographs supported that mechanical anisotropy reflected the orientation of collagen fibers.

\section{Orientation Patterns along the Transverse Direction}

Figure 5 shows the values of $\mathrm{MW}_{(\mathrm{TD} / \mathrm{CCD})}$ at 12 $\mathrm{GHz}$ and the orientation patterns at five different positions along the TD of sample No. 10. The positions of No. 10 (-32.5) and No. 10 (32.5) are near the dorsum. $\beta$, DFO and $\mathrm{MW}_{(\mathrm{TD} / \mathrm{CCD})}$ are $17^{\circ}, 1.174$ and 1.160 , respectively, at the position of No. 10 (32.5).

The orientation angles at five different positions of sample No. 10 are relatively small, ranging from $0^{\circ}$ to $17^{\circ}$. The small orientation angles suggest that collagen fibers were, on average, aligned parallel to CCD. DFO was largest near the center of the dorsum and decreased with the changing position from the dorsum to the abdomen. Also, $\mathrm{MW}_{\text {(TD/CCD) }}$ was large near the

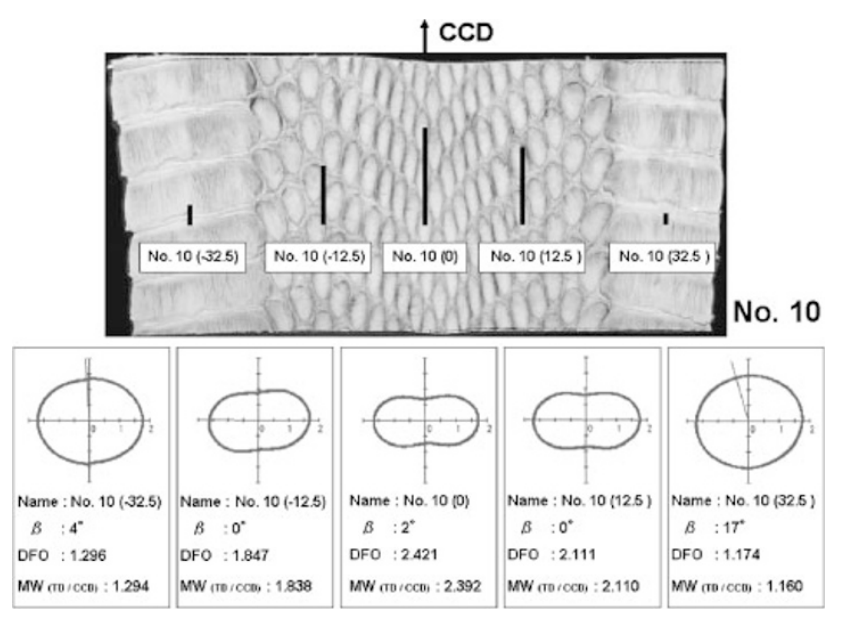

Figure 5. The angular dependence of transmitted microwave intensity at $12 \mathrm{GHz}$ and the $\mathrm{MW}_{(\mathrm{TD} / \mathrm{CCD})}$ at five different positions along the TD for sample No. 10. $\beta$, DFO and $\mathrm{MW}_{(\mathrm{TD} / \mathrm{CCD})}$ is, respectively, the orientation angle, degree of fiber orientation and ratio of transmitted microwave intensity in TD and CCD. The lengths of the line segments shown in Figure 5 represent the value of $\left(\mathrm{MW}_{(\mathrm{TD} / \mathrm{CCD})}-1.0\right)$, which reflects the net degree of transmitted microwave intensity. Line segment lengths are an arbitrary scale.

center of the dorsum and also decreased with the changing position from the dorsum to the abdomen. The value of DFO is similar to that of $\mathrm{MW}_{(\mathrm{TD} / \mathrm{CCD})}$ when $\beta$ is very small. Thus, $\left(\mathrm{MW}_{(\mathrm{TD} / \mathrm{CCD})}-1.0\right)$ is used as the index reflecting anisotropy in two perpendicular directions of TD and CCD instead of (DFO $-1.0)$ only when $\beta$ is very small.

The value of $\left(\mathrm{MW}_{(\mathrm{TD} / \mathrm{CCD})}-1.0\right)$ is shown by the length of the line segment in Figure 5. This result indicates that the skin shows strong anisotropy in the dorsum, while it shows weak anisotropy in the abdomen.

\section{Orientational Distribution of Collagen Fibers}

Figures $6 \mathrm{a}, 6 \mathrm{~b}$ and $6 \mathrm{c}$ show the direction, $\beta$, and the degree of fiber orientation, DFO, determined at different positions with intervals of $2.5 \mathrm{~mm}$ using Osaki's microwave method, and the orientation patterns obtained at three different positions along the TD for sample Nos. 10, 31 and 1, prepared from the trunk, caudal and cranial regions of cobra skin, respectively. The direction and the net degree of fiber orientation are expressed by the inclination and length of a line segment in Figures $6 a, 6 b$ and $6 c$, respectively.

With respect to sample Nos. 10 and 31, the direction of fiber orientation was, on average, parallel to the CCD, while the degree of fiber orientation increased gradually with the changing position from the abdomen to the dorsum, and was largest at the center 
(a)

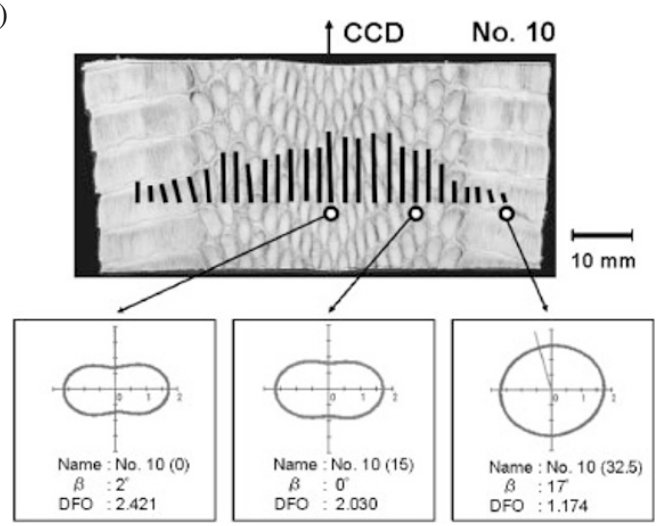

(b)

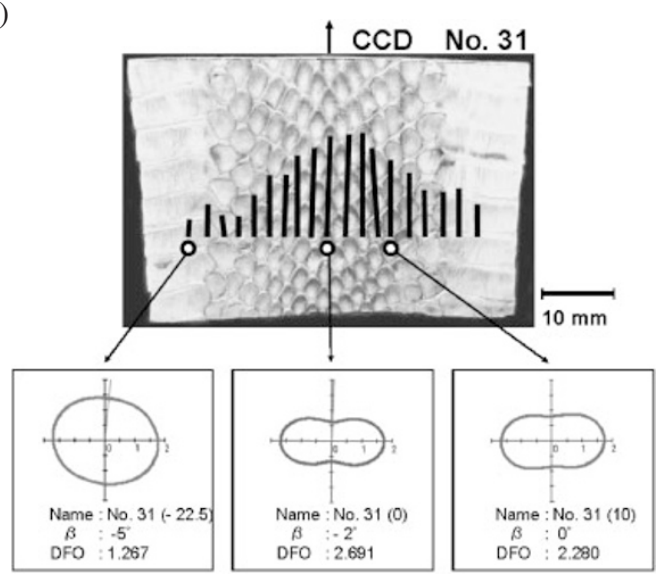

(c)

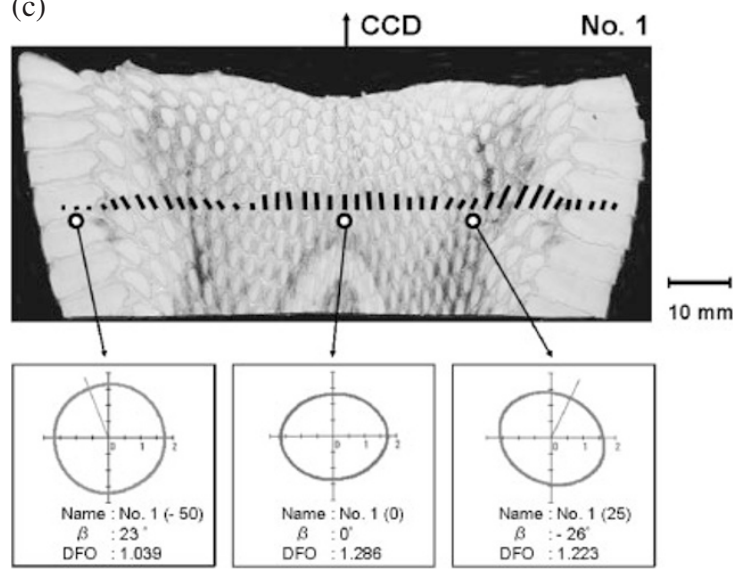

Figure 6. $6 a, 6 b$ and $6 c$ show the direction and degree of fiber orientation determined using the microwave method at each interval of $2.5 \mathrm{~mm}$ along the TD for sample Nos. 10, 31 and 1, respectively. Sample Nos. 10, 31 and 1 were prepared from the trunk, caudal and cranial regions, respectively. The length and inclination of the line segments shown in Figures $6 \mathrm{a}, 6 \mathrm{~b}$ and $6 \mathrm{c}$ represent the net degree of fiber orientation and the orientation angle, respectively. a: The orientation of collagen fibers in the trunk region and the orientation patterns at $12 \mathrm{GHz}$ at position Nos. $10(0), 10$ (15) and 10 (32.5). b: Orientation of collagen fibers in the caudal region and the orientation patterns at $12 \mathrm{GHz}$ at position Nos. 31 $(-22.5), 31(0)$ and 31 (10). c: Orientation of collagen fibers in the cranial region and orientation patterns at $12 \mathrm{GHz}$ at position Nos. 1 $(-50), 1(0)$ and $1(25)$.

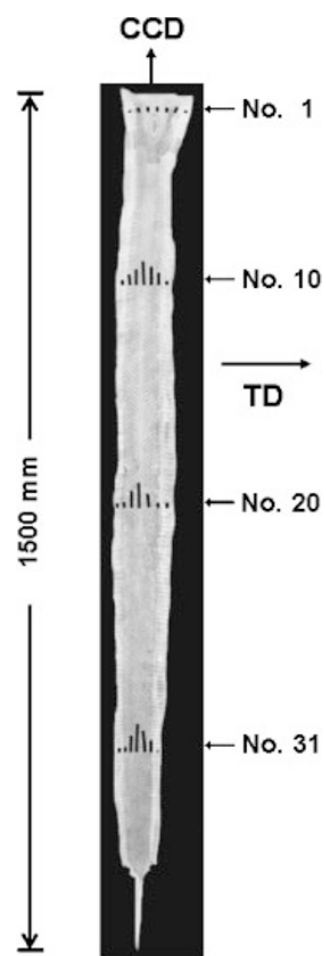

Figure 7. The orientational distribution of collagen fibers in a whole cobra skin. The length and inclination of the line segments represent the net degree of fiber orientation and the orientation angle, respectively.

of the dorsum in the trunk and caudal regions. The orientation patterns in the dorsum showed an egg cocoon shape, whereas those in the abdomen $\{$ e.g., sample Nos. 10 (32.5) and $31(-22.5)\}$ were almost circular, as shown in Figures $6 \mathrm{a}$ and $6 \mathrm{~b}$. The obtained orientation patterns show that the skin in the dorsum has strong anisotropy, whereas the skin in the abdomen has weak anisotropy.

On the other hand, the direction of fiber orientation changed markedly in three different directions along the TD of the cranial region (sample No. 1), and the degree of fiber orientation was very small around the cranial region, on average, relatively small compared with that in other regions. The orientation patterns shown in Figure 6c were almost circular. These results show that cranial skin has only weak anisotropy in its circumference.

Figure 7 shows the orientational distribution of collagen fibers in a whole cobra skin. The skin shows strong anisotropy in the trunk and caudal regions, closely suggesting marked anisotropic motion, while it shows weak anisotropy in the abdomen, suggesting isotropic expansion. In particular, the cranial skin seems to expand in all directions when swallowing prey. 


\section{DISCUSSION}

It is impossible to measure mechanical anisotropy in small regions of cobra leather; however, Osaki's microwave method has made it possible to determine the fiber orientation of collagens at different positions with intervals of $2.5 \mathrm{~mm}$ by discretely shifting the position of the cobra leather. Thus, the present study showed that Osaki's microwave method could be used to determine collagen-fiber orientation, even in restricted regions, instead of the mechanical method, and gave the orientational distribution of collagen fibers in the whole skin of a cobra.

The orientation patterns in the trunk and caudal regions suggest that cobra skin moves in a markedly anisotropic direction. The weak anisotropy in the abdomen suggests that the abdomen expands and shrinks easily in all directions when moving and swallowing. On the other hand, the orientation patterns in the cranial region suggest that the skin expands equally in all directions because the cobra mouth has to expand as much as possible when eating.

The strong anisotropy in the skin around the circumference of the cobra body seems to be closely related to functional motion during locomotion and when swallowing prey.

The orientational distribution of collagen fibers in the whole cobra skin will provide important information for understanding the details of functional motion, such as shrinkage and expansion, of the cobra.

\section{REFERENCES}

1. A. Bigi, A. Ripamonti, and N. Roveri, Connect. Tissue Res., 5, 37 (1977).

2. M. Green, D. H. Isaac, and G. M. Jenkins, Biomaterials, 8, 427 (1987).

3. X. Markenscoff and I. V. Yannas, J. Biomech., 12, 127 (1979).

4. T. L. Foutz, E. A. Stone, and C. F. Jr. Abram, Am. J. Vet. Res., 53, 788 (1992).

5. L. E. Edsberg, R. E. Mates, R. E. Baier, and M. Lauren, J. Rehabil. Res. Dev., 36, 133 (1999).

6. J. M. McMahon, A. Boyde, and T. G. Bromage, Anat. Rec., 242, 147 (1995).

7. R. B. Martin, S. T. Lau, P. V. Mathews, V. A. Gibson, and S. M. Stover, J. Biomech., 29, 1515 (1996).

8. K. Niitsuma, S. Miyagawa, and S. Osaki, Eur. J. Morphol., 42, 193 (2005).

9. S. Osaki, Tappi J., 70, 105 (1987).

10. S. Osaki, Tappi J., 72, 171 (1989).

11. K. Yamamoto, S. Osaki, S. Yamashita, and M-o. Yamada, Cell. Mol. Biol., 34, 571 (1988).

12. S. Osaki, M-o. Yamada, A. Takakusu, and K. Murakami, Cell. Mol. Biol., 39, 673 (1993).

13. S. Osaki, Anat. Rec., 254, 147 (1999).

14. S. Osaki and T. Ohashi, Cell. Mol. Biol., 50, 559 (2004).

15. S. Osaki, S. Tohno, Y. Tohno, K. Ohuchi, and Y. Takakura, Anat. Rec., 266, 103 (2002).

16. K. Ohuchi, S. Osaki, S. Tohno, Y. Tohno, Y. Takakura, and S-i Kikuchi, Cell. Mol. Biol., 49, 425 (2003).

17. S. Osaki, J. Appl. Phys., 67, 6513 (1990).

18. S. Osaki, Nature, 347, 132 (1990).

19. S. Osaki, Rev. Sci. Instrum., 68, 2518 (1997).

20. S. Osaki, Polym. J., 19, 821 (1987). 